\title{
Estimation of X-mode reflectometry first fringe frequency using neural networks
}

\author{
D.E. Aguiam*, A. Silva*, L. Guimarãis*, P.J. Carvalho*, G.D. Conway ${ }^{\dagger}$, B. Goncalves*, L. Meneses*, J.M. \\ Noterdaeme $^{\dagger \ddagger}$, J.M. Santos*, A.A. Tuccillo ${ }^{\S}$, O. Tudisco ${ }^{\S}$, ASDEX Upgrade Team ${ }^{\dagger}$ \\ *Instituto de Plasmas e Fusão Nuclear, Instituto Superior Técnico, Universidade de Lisboa, 1049-001 Lisboa, \\ Portugal \\ ${ }^{\dagger}$ Max-Planck-Institut für Plasmaphysik, Boltzmannstr. 2, D-85748 Garching, Germany \\ ${ }^{\ddagger}$ Ghent University, Applied Physics Department, B-9000 Gent, Belgium \\ ${ }^{\S}$ ENEA, Dipartimento FSN, C. R. Frascati, via E. Fermi 45, 00044 Frascati (Roma), Italy
}

\begin{abstract}
One of the main challenges in X-mode reflectometry is the correct determination of the group delay measurement used for density profile reconstruction. The $X$-mode upper cut off group delay measurement can be used to reconstruct the electron density profiles from the near zero density. However, due to the broad operational conditions of experimental fusion devices, the start of the upper cut off region can occur at any probing frequency. The first fringe of the interference signal measured by reflectometry, that corresponds to the start of the upper cut off reflection is used together with the magnetic field profile to determine vacuum distance between the reflectometer antenna and the start of the plasma. An incorrect estimation of the first fringe probing frequency not only introduces a radial error but also a group delay error, affecting the shape of the resulting density profile. In this work we present the new developments in the automatic first fringe estimation required for the reliable reconstruction of density profiles, used in the multichannel $\mathrm{X}$-mode density profile reflectometry diagnostic recently installed on ASDEX Upgrade. An improved algorithm to estimate and track the frequency of the first fringe along a discharge is introduced. Tests show that it is able to correctly determine the first fringe for most discharges. However, for a number of unanticipated cases, the algorithm provides jitter and imprecise results, introducing errors in the reconstructed density profiles. We also present a novel neural network approach for the first time for the estimation of the first fringe frequency. A comprehensive training set was carefully selected by experienced reflectometry diagnosticians and used to train the neural network model using the open source software libraries TensorFlow and Keras. The resulting neural network is able to provide more precise first fringe estimations than the previous algorithm. The reconstructed density profiles, using both algorithms, are presented and compared.
\end{abstract}

Index Terms-X-mode reflectometry, first fringe estimation, first cut off reflection, neural networks

\section{INTRODUCTION}

A new multichannel $\mathrm{X}$-mode reflectometry diagnostic to measure the edge electron density profiles was recently installed on ASDEX Upgrade. This diagnostic has three channels embedded in the ICRF antenna to probe the facing edge plasma at the top, middle and bottom poloidal regions of the radiating surface [1]. It aims to contribute to ICRF operation studies, such as the study of ICRF power coupling to the plasma [2], which require millimeter resolution in the radial density profile.
In reflectometry, the electron density profile is reconstructed from the group delay measurements of probing waves with varying frequency after reflection at the corresponding plasma density cut off layers [3]. X-mode propagation in magnetized plasmas is characterized by two cut off regions that depend on the local plasma density and magnetic field. The use of the upper cut off region, and the knowledge of the imposed magnetic field profile along the line of sight, allow for the measurement of density profiles from the edge density up to the core.

A probing wave is launched from the magnetic low field side of the vessel, propagating through a small vacuum distance, and through the plasma up to a reflection layer. The cut off frequency, at which the wave is reflected back to the receiving antenna, depends on the local electron density and magnetic values. The upper $\left(f_{u c}\right)$ and lower $\left(f_{l c}\right)$ cut off frequencies are determined by:

$$
\begin{gathered}
f_{u c}=\sqrt{f_{p e}^{2}+\frac{f_{c e}^{2}}{4}}+\frac{f_{c e}}{2}, \\
f_{l c}=\sqrt{f_{p e}^{2}+\frac{f_{c e}^{2}}{4}}-\frac{f_{c e}}{2},
\end{gathered}
$$

where $f_{p e}^{2}(r)=n_{e}(r) e^{2} /\left(4 \pi \epsilon_{0} m_{e}\right)$ is the electron plasma frequency, dependent on the electron density $n_{e}$, and $f_{c e}(r)=e B(r) /\left(2 \pi m_{e}\right)$ is the electron cyclotron frequency, dependent on the local magnetic field $B$.

Density profile reconstruction depends on the correctness of the magnetic field profile estimation, the group delay measurement and the method of reconstruction. In our procedure, the magnetic field information is provided by the equilibrium codes on ASDEX Upgrade and the reconstruction method is described in [3]. The probing frequency at which the first reflection occurs is called the First Fringe (FF) frequency and indicates the start of the group delay measurement of the X-mode upper cut off reflection. This first reflection occurs when the plasma electron density is $n_{e} \approx 0$, and when the propagating wave's frequency matches the local electron cyclotron frequency limit $f=f_{c e}^{+}$, as determined from (1). The group delay measurement starts at this FF frequency and is proportional to the beat frequency of the 

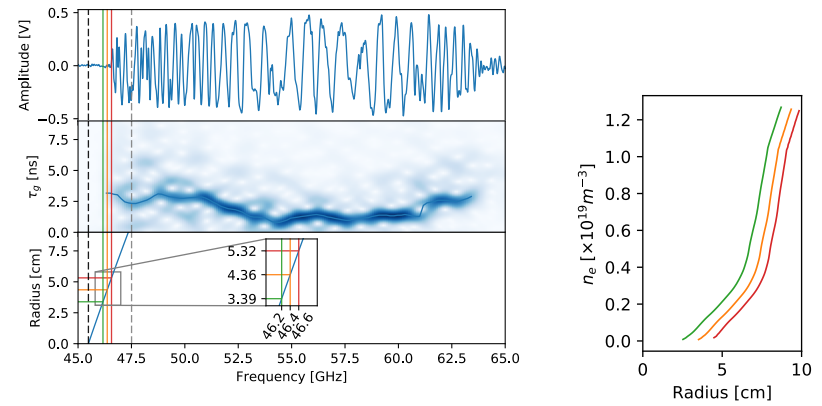

Fig. 1. Influence of small FF estimation shifts in resulting density profile. Left top and middle are the acquired raw signal and resulting group delay spectrogram. Bottom is the radial profile of the electron cyclotron frequency along the line of sight with origin at the launching antenna's mouth. On the right are the reconstructed density profiles corresponding to the group delay measurements starting at each of the FF frequencies indicated on the left.

acquired signal divided by the probing frequency variation rate $\tau_{g}=f_{b}(d t / d f)$.

The FF frequency together with the knowledge of the magnetic field profile are used to determine the vacuum distance between the launching antenna and the start of the plasma. Errors in the vacuum distance estimation result in radial shifts of the reconstructed plasma density profile, along the line of sight of the antenna. In addition, the incorrect determination of the propagation group delay affects the density profile shape, as the error is integrated along the profile reconstruction

Figure 1 shows three possible FF estimations and their respective processed density profiles. The FF frequencies have a spacing of $200 \mathrm{MHz}$, which result in density profile radial variations of up to $9.5 \mathrm{~mm}$. This indicates that a relatively small error in FF estimation (up to \pm 3 probing frequency points of the spectrogram, or less than $1 \%$ of the probing bandwidth) may result in large radial shifts of the density profile. It is important to note that probing frequency resolution depends on the spectrogram window (214 samples correspond to a $2 \mathrm{GHz}$ window) and step (10 samples, around $93.5 \mathrm{MHz}$ per step) parameters, determined for best group delay determination performance.

In this work we present the new developments introduced in the determination of group delay measurement in the ICRF embedded reflectometer on ASDEX Upgrade. We first introduce the difficulties involved in the development of automatic $\mathrm{X}$-mode upper cut off first fringe estimation algorithms able to robustly operate in all operational conditions. We describe the ampfilt algorithm, which is based on existing published algorithms and capable of determining the FF frequency even in the presence of lower cut off reflection signal. We finally introduce a novel neural network approach to estimate the FF frequency from the group delay spectrogram aiming to provide better FF estimation performance than previous estimation methods. The errors produced by both estimation methods are evaluated over a large experimental dataset validated by experienced human diagnosticians. The performance of both approaches is also evaluated by comparing the precision of their FF estimation evolution over a complete discharge and by comparing the reconstructed density profiles

\section{Automatic estimation of the X-Mode First FRINGE FREQUENCY}

In the ideal scenario for X-mode upper cut off measurements, there is no plasma reflection signal up to first upper cut off, where the signal amplitude distinctly increases. This can be observed in Figure 2 (left), where the acquired raw signal and resulting spectrograms for different operational conditions of the diagnostic on ASDEX Upgrade are shown.

The probing frequency at which the signal amplitude's gradient suddenly increases can be interpreted as the FF frequency and the start of the upper cut off plasma reflection. This approach has been used in many different devices such as DIII-D [4], Tore Supra [5], EAST [6]. Amplitude based methods require a high dynamic range of the diagnostic measurement and assume that the plasma reflection signal power is consistently higher than other reflections, such as back wall reflection, spurious noise or lower cut off reflection.

More advanced techniques, such as the ones used at JET [7], Alcator C-Mod [8], DIII-D [9], contemplate both the frequency response and amplitude of the reflectometry signal. Such approaches provide additional reliability by ignoring broadband spurious signals that may occur before the expected reflection signal at the plasma edge.

The FF search range may be reduced by using the magnetic field profile along the line of sight of the antenna, provided by the magnetic equilibrium. Considering that the probing waves propagate along the line of sight with origin at launching reflectometry antennas, and assuming that there is no plasma behind the antennas, since these are embedded behind the ICRF antenna limiters, then the first upper cut off plasma reflection must occur somewhere along this line of sight where the probing wave frequency matches the local electron cyclotron frequency $\left(f_{F F} \approx f_{c e}\left(r_{F F}\right)\right)$. As the magnetic field increases along the line of sight, then $f_{F F}$ must be higher than the cyclotron frequency at the origin of the line of sight $f_{\text {wall }}$ and lower than $f_{\text {sep }}$, the cyclotron frequency at the separatrix position $\left(r_{s e p}\right.$, which is also obtained from the magnetic equilibrium), where the plasma must exist.

In the analysis of the reflectometry raw data presented in previous work [10], we showed how the increase of the plasma core electron density results in a lower cut off reflection appearing into the 40-68 GHz probing frequency window. The typical group delay measurements in such high plasma core density conditions can be observed in Figure 2 (right). The consistently high amplitude of the lower cut off reflection signal typically breaks amplitude-based FF estimation algorithms, which can not easily distinguish between upper and lower cut off regions. Over half of the plasma discharges at ASDEX Upgrade acquired during the commissioning phase of the diagnostic in 2016 meet the high core density conditions for the appearance of this lower cut off reflection in the 40$68 \mathrm{GHz}$ probing window sometime during the discharge. For this reason, it is crucial that any FF estimation algorithm be robust enough to estimate the FF in both the simpler upper cut off conditions but also in the scenarios with presence of both upper and lower cut off reflections. 


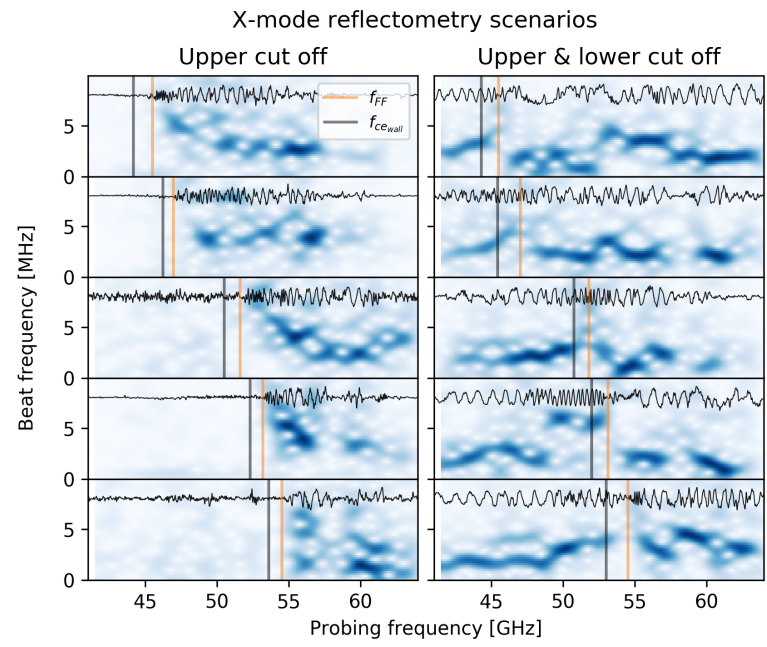

Fig. 2. Different typical X-mode reflectometry group delay spectrogram measurements with only upper cut off (left) and both upper and lower cut off (right). The latter is a typical result for high core density plasmas.

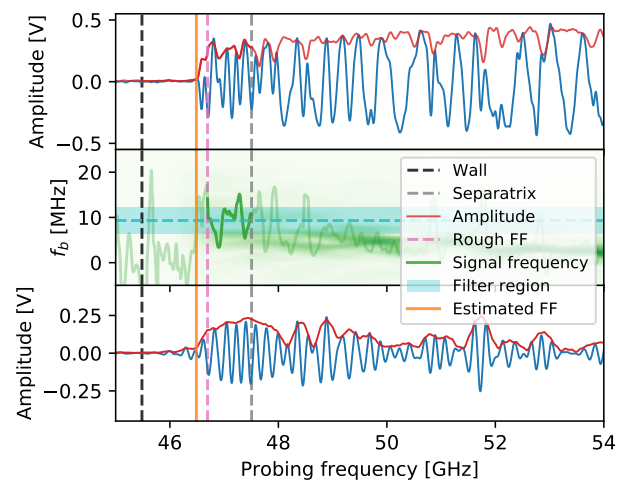

Fig. 3. FF estimation steps using the ampfilt algorithm for a reflectometry raw signal with only $\mathrm{X}$-mode upper cut off reflection.

\section{AMPFILT FF ESTIMATION ALGORITHM}

In the development of the new X-mode density profile reflectometry diagnostic we implemented the ampfilt algorithm, adapted from the first fringe estimation algorithm from DIII-D presented in [9].

In this algorithm, a rough FF frequency $f_{\text {rough }}$ is estimated from the highest amplitude gradient in the bounded $\left[f_{\text {wall }}, f_{\text {sep }}\right]$ range. The average frequency of the reflectometry interference signal in the range $\left[f_{\text {rough }}, f_{\text {sep }}\right]$ is calculated and a bandpass filter around this frequency filters the original signal. The FF is then taken at the frequency for which the filtered signal amplitude rises above a certain threshold. Figure 3 shows the sequence of the ampfilt algorithm and a good estimation of the FF frequency in a scenario with only $\mathrm{X}$-mode upper cut off reflection.

In addition, the ampfilt algorithm detects if there is a lower cut off reflection signal and switches to a second FF estimation procedure. The presence of the lower cut off refelction is detected when the average signal amplitude to the left of $f_{\text {wall }}$ is above 0.8 times the average signal amplitude to the right of

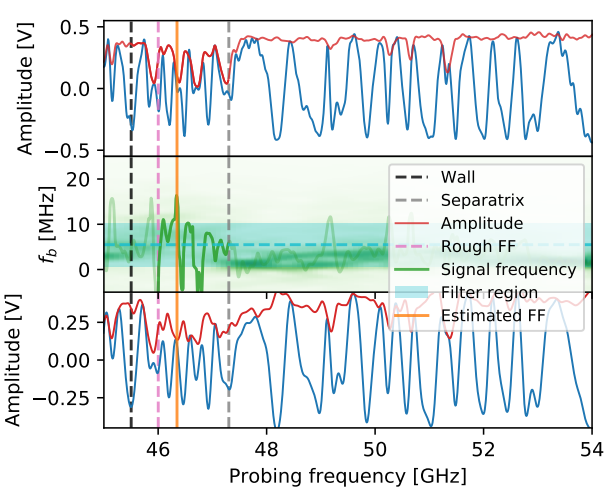

Fig. 4. FF estimation steps using the ampfilt algorithm for a reflectometry raw signal of a high core density plasma, resulting in both X-mode upper and lower cut off reflections.

$f_{\text {sep }}$. In theory, the FF reflection occurs immediately after the absorption layer where $n_{e}=0$ and $f=f_{c e}$. In this vicinity above the cut off, the probing wave is slowed down resulting in a high group delay measurement. In the presence of the lower cut off, the reflection beat frequency signals are consistent on both sides of FF and the FF is estimated to occur around the peak of the beat frequency, where the signal amplitude is also low. In Figure 4 the ampfilt estimates the FF frequency in a high core electron density plasma resulting in the presence of a lower cut off reflection in the probing window. As seen, the FF estimation coincides with an $f_{b}$ peak and a dip in reflection signal amplitude.

The ampfilt algorithm provides a reliable FF estimation in most situations. However, the algorithm may have large unexpected variation of the FF frequency estimation for consecutive measurements in a discharge that are not correlated to any known plasma event or noise source. This estimation imprecision results in sporadic radial shifts of the reconstructed density profiles, which do not correspond to real profile features. These erroneous density profiles must then be detected and removed.

\section{NEURAL NETWORK FOR FIRST FRINGE ESTIMATION}

Generally, an experienced human diagnostician analysing the different group delay spectrograms can detect certain patterns that give hints on where the start location of the upper cut off reflection might be. These may be combinations of frequency peak evolution, amplitude dynamics and knowledge of the $f_{\text {wall }}$ and $f_{\text {sep }}$ probing frequency delimiters. However, translating these cues into discriminating rules and programming these into explicit algorithms is not an easy task due to the effects of the broad range of operational conditions has on measured signals, such as magnetic field, plasma electron density, noise due to different heating power sources, etc.

Neural networks $(\mathrm{NN})$ are computational methods used ubiquitously in machine learning systems. These networks consist of several layers of neurons, interconnected by weighted links, that can be trained to mimic the pattern recognition procedures of humans. These techniques are used 
to recognize objects, classify images, transcribe and translate speech, provide accurate web search results, make financial market predictions, etc [11].

With the increasing popularity of machine learning, several tools to create, train and use neural network models, have been created. Google has open sourced its versatile TensorFlow [12] tool to express machine learning algorithms. Keras [13], [14] is a deep learning Python library interface that can work with the TensorFlow or other machine learning backends. These open source initiatives greatly reduce the barrier to entry and eased the learning curve needed to implement machine learning techniques in new fields of application.

Neural networks models have also been used in fusion research to replace existing computationally complex reconstruction algorithms. A well trained NN model may be capable of reproducing the same results as an explicit algorithm, within acceptable accuracies, while sometimes being faster to compute than their original counterparts. NN applications in fusion research include the tomographic reconstruction of bolometry data [15], real-time plasma impurity monitoring [16] and realtime evaluation and reconstruction of reflectometry density profiles for plasma position control [17], [18]. In addition, the pattern recognition capabilities of neural networks have also been used to classify and predict tokamak disruptions [19], [20].

We present a new neural network approach aimed to improve the precision of the first fringe frequency estimation, when compared with the ampfilt algorithm, by mimicking the pattern recognition of human diagnosticians looking at reflectometry spectrogram results. The supervised training of the NN uses a large comprehensive training dataset. This training dataset was created by experienced X-mode reflectometry diagnosticians using experimental data acquired by the ICRF embedded X-mode reflectometer during the 2016 ASDEX Upgrade campaign.

\section{A. Creation of the training dataset}

The first essential step to create a neural network, and train it using supervised learning, is to create a large dataset of train and test cases with good enough variability so that the trained $\mathrm{NN}$ is capable of predicting the correct answer in most cases. A total of 695 discharges were acquired with the new X-mode reflectometry diagnostic during the 2016 ASDEX Upgrade campaign. The distribution of these discharges depending on their magnetic field and core electron density operational parameters is shown in Figure 5.

The lower and upper magnetic field limits indicate the operational limits of the reflectometry diagnostic to observe the start of the upper cut off region inside the 40-68 GHz probing frequency band. Most discharges on ASDEX Upgrade have a magnetic field of around $2.5 \mathrm{~T}$. A higher core plasma electron density indicates that the lower cut off reflection may also appear inside the probing band window sometime during the discharge. The core density threshold curve (dashed curve in Figure 5) indicates discharges where the lower cut off reflection signal appears in the probing band and interferes with the upper cut off reflection signal.

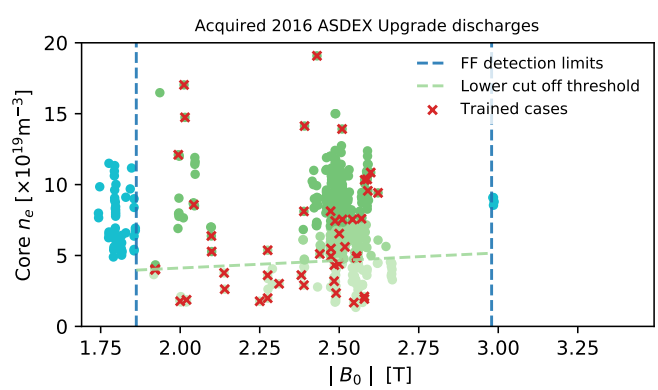

Fig. 5. Distribution of the plasma discharges depending on their magnetic field and core electron density acquired during the 2016 ASDEX Upgrade campaign.

The training dataset consists of the raw data, operational conditions and the selected first fringe frequency of 2209 raw individual reflectometry acquisitions. These individual cases were selected from 47 distinct discharges dispersed along the measurable operational range (represented by $\times$ in Figure 5). A graphical tool was developed to plot the raw data, group delay spectrogram and relevant information such as signal amplitude, $f_{\text {wall }}$ and $f_{\text {sep }}$, aiding the diagnostician in selecting the correct FF frequency. It is important to note that the $\mathrm{NN}$ is trained to be as good as the experienced diagnostician, which, nevertheless, result in a certain degree of uncertainty. The training cases were also selected with different heating powers (14.2\% of the cases occurring with ICRH power, $56.6 \%$ with ECRH and $34.3 \%$ with NBI), during ELM events (2.6\% of the cases) and with lower cut off reflection inside the probing window $(24.5 \%)$.

\section{B. Preprocessing and extending the dataset}

The dataset contains the raw data, time and magnetic information and estimated "true" FF values for each of the 2209 cases. The NN interprets the calculated spectrogram data and estimates the corresponding FF frequencies from it. Prediction performance can be increased by defining a region of interest (ROI) around the expected FF position and retaining only this data as input to the NN. In a first step, the spectrogram data is restricted to the $0-25 \mathrm{MHz}$ beat frequency region, since there is no useful reflection data outside this range, by design, even though the total spectrogram spans $\pm 100 \mathrm{MHz}$. Then, the search region of interest (ROI) is delimited by $f_{\text {wall }}$ and $f_{\text {sep }}$, within which the FF must be located, as shown in Figure 6. The data outside the ROI is zeroed out, resulting in the preprocessed input spectrogram image shown in Figure 7. The true FF frequency value is discretized into a one-hot vector ${ }^{1}$ with 1120 possible discrete values between $40 \mathrm{GHz}$ and $64 \mathrm{GHz}$ (signal quality decreases above $64 \mathrm{GHz}$ ), resulting in $21 \mathrm{MHz}$ FF frequency resolution versus the original $93.5 \mathrm{MHz}$ probing frequency resolution of the spectrogram. There is a trade off between acceptable discretization resolution and FF coverage of the training dataset. A higher resolution would require a larger, more comprehensive training set.

\footnotetext{
${ }^{1} \mathrm{~A}$ one-hot vector is a sequential vector with 1 at the single position corresponding to the true value and $0 \mathrm{~s}$ in the remaining positions.
} 


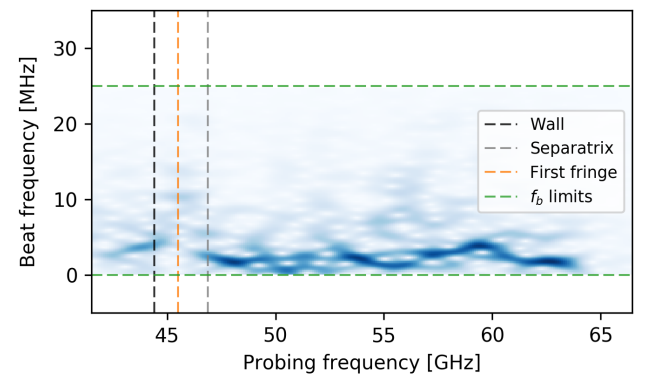

Fig. 6. Raw X-mode reflectometry spectrogram data with both upper and lower cut off reflection data. Horizontal lines are the considered upper and lower beat frequency limits. Vertical lines are the $f_{\text {wall }}$, true $f_{F} F$ and $f_{\text {sep }}$.

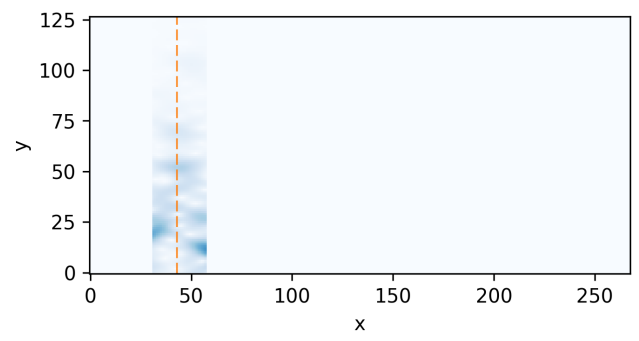

Fig. 7. Preprocessed 2D spectrogram image used as input for the neural network and respective true FF location

The neural network performance is inherently dependent on the quality and size of the training dataset. NN predictions are limited to the trained cases and our sparse dataset does not cover all the possible discretized FF values. This sparsity increases prediction error for inadequately trained FF cases. A broader training set is required to improve prediction performance.

Thus, the existing preprocessed dataset was extended by assuming that the spectrogram signatures in the ROI are similar for FF frequencies close to each other. Since the spectrogram data outside the ROI is zeroed out, the ROI may be shifted sideways and assume that it corresponds to a new case where the FF is equally shifted. Using these assumptions we were able to create an extended dataset by shifting each of the original dataset case several times within its $\pm 1 \mathrm{GHz}$ vicinity (each spectrogram window spans $2 \mathrm{GHz}$ ). The dataset was extended from 2209 to 112659 total new cases, providing a much better FF coverage.

\section{Definition and training of the neural network model}

In our approach, a 2D convolutional neural network model interprets the spectrogram data to determine the start of the upper cut off reflection in a similar way to a human diagnostician. This network receives the input data represented in Figure 7 and outputs the likelihood distribution of the FF along the 1120 discrete FF values. The highest probability is then assumed as the first fringe frequency.

The neural network model was defined using the Keras Python library and trained with the TensorFlow backend. The

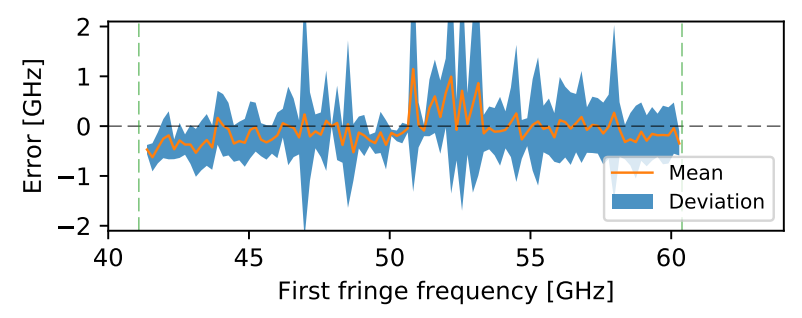

Fig. 8. Mean and standard deviation distribution error of the ampfilt algorithm using the known sparse dataset.

input layer of the neural network model is a $2 \mathrm{D}$ convolutional layer with $127 \times 268$ input grid shape, $505 \times 5$ filters, rectified linear unit (ReLu) activation and stride 3. A 2x2 pooling layer was used to down sample the data which was then flattened. Then there is a fully-connected (FC) layer with ReLu activation and 1024 elements, followed by the output FC layer with sigmoid activation and 1120 output shape. The network was compiled and trained using a categorical cross entropy loss function and Adam optimization [21].

In order to correctly validate the neural network training and prevent over fitting to the training data, it is necessary to separate the dataset into training and validation subsets. This was achieved by randomly splitting $90 \%$ of the dataset for training and the remaining for validation, keeping the same FF distribution in both datasets.

\section{EXPERIMENTAL RESULTS}

Here we analyse the ampfilt and neural network based FF estimation algorithms over the dataset of known FF values. The differences in FF coverage and the errors of the sparse and extended datasets are explained. We then use the both algorithms to estimate the FF frequency along the same discharge and compare the reconstructed density profiles.

\section{A. Analysis of the ampfilt estimation performance}

The ampfilt was tested using the sparse dataset created for the training of the neural network. The FF frequency was estimated for all the cases and the errors were produced calculating the difference to the corresponding registered dataset FF value. The error distribution is obtained by discretizing the FF frequency axis into a smaller number of bins and and calculating the average and standard deviation errors within these bins. The mean and standard deviation error distribution of the ampfilt algorithm are shown in Figure 8. Over the whole range, the ampfilt algorithm has FF estimation error average of $-80 \mathrm{MHz}$ and a standard deviation of $790 \mathrm{MHz}$. However, error peaks of over $1 \mathrm{GHz}$ may also occur, consequently resulting in large radial and shape errors of the density profile. The small average negative error bias is considered a systematic error specific to this dataset and is not compensated since it falls within \pm 1 point of the spectrogram probing frequency resolution. 


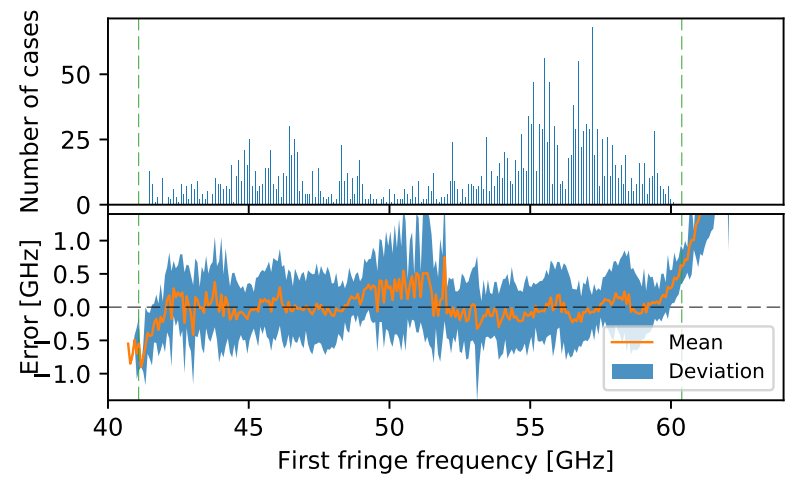

Fig. 9. First fringe distribution of the sparse dataset used to train the neural network and resulting error distribution.

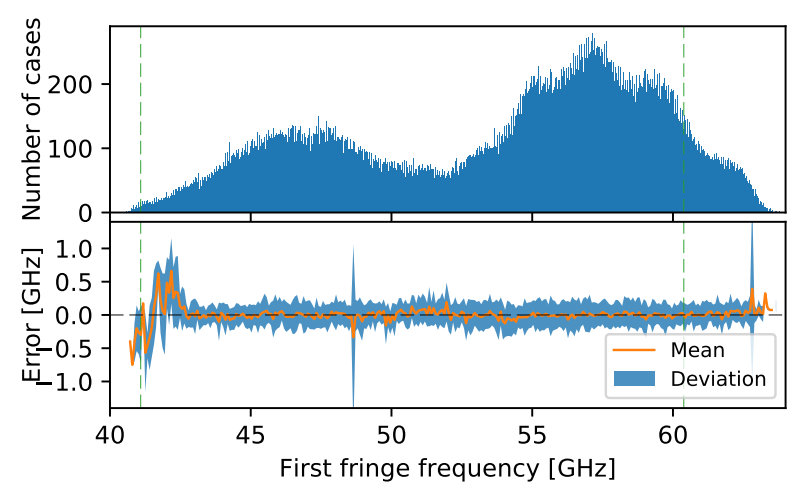

Fig. 10. First fringe distribution of the extended dataset used to train the final neural network and resulting error distribution.

\section{B. Analysis of the neural network training and estimation}

The FF frequency distributions of the sparse and extended datasets are represented in Figure 9 (top) and Figure 10 (top), respectively. As can be observed, the sparse dataset does not contain all of the possible FF frequency values, while the extended dataset provides data for the a much better FF coverage.

Two neural network models were trained using the training subsets of the sparse and extended datasets. The corresponding errors were calculated using the more comprehensive extended validation subset $(10 \%$ of the extended dataset). The sparse and extended NN FF estimation error distributions are represented in Figures 9 (bottom) and 10 (bottom), respectively.

The sparse NN model can not extrapolate correctly to cases outside its trained range, resulting in the observed error increase near the outer edges of the validation range. To avoid error biasing of the extrapolated results, the FF estimation error is calculated within the limits of the original sparse training universe, represented by the vertical dashed lines. The FF estimation error of the network trained with the sparse dataset has an average of $30 \mathrm{MHz}$ and an error deviation of $550 \mathrm{MHz}$. This high deviation can be attributed to the sparsity of the training dataset and since the extended validation dataset includes many untrained FF cases. The neural network should be trained with multiple different input cases for the same FF value, which is not correctly achieved using this sparse dataset.

However, training the neural network model with the extended dataset reduced the average FF estimation error to $-0.5 \mathrm{MHz}$ and the error standard deviation to $230 \mathrm{MHz}$, within the same validations limits. This is due to a much better individual FF coverage of the extended training dataset (there is a higher number of cases for each of the discrete FF values) due to the overlap of the shifted ROI cases. However, the increased error near the lower limit of the FF range can be attributed to a lower coverage of the training universe in this region, when compared with remaining dataset. The average error stabilizes close to zero when there are more than 40 different training cases per FF value.

\section{Comparison of reconstructed density profiles}

The density profile evolutions of discharge \#33841 of ASDEX Upgrade were reconstructed using the ampfilt algorithm and the extended neural network model, as shown in Figure 11. We use the NN model trained with the extended dataset as it provides a more constant error deviation response than the sparse NN model, as well as better extrapolation near the outer edges. A high core electron density, above approximately $6 \times 10^{19} \mathrm{~m}^{-3}$, resulted in the lower cut off reflection appearing inside the probing window during the 1.2-4.2 seconds interval (shaded region in top plot). A $2 \mathrm{~cm}$ outer radial scan of the separatrix position occurred at around 3 seconds. The core magnetic field varied from $-2.47 \mathrm{~T}$ up to $-2.54 \mathrm{~T}$, as can be observed by the corresponding cyclotron frequency evolution at the wall, $f c e_{\text {wall }}$.

The FF frequency estimations using the ampfilt and NN approaches are shown in the second plot. The $\mathrm{FF}_{\text {ampfilt }}$ result has higher variation between consecutive time steps. This jitter is increased in the presence of the lower cut off reflection. However, the neural network estimation, $\mathrm{FF}_{N N}$, has a better precision than $\mathrm{FF}_{\text {ampfilt }}$ throughout the discharge, even during lower cut off reflection.

A Kalman filtering technique [22] is used to increase the precision of the FF estimation used for each density reconstruction. The filtered FF value is predicted for each time step, from the previous filter state and the new FF estimation, based on the idea that the vacuum distance should not vary much between two consecutive acquisitions $(\approx 100 \mu s)$. This filter greatly reduces the FF estimation jitter of either algorithm, enabling a more accurate density profile reconstruction.

The reconstructed density profiles using both algorithms are represented at the bottom of Figure 11. The positions of the density profile are referenced as the distance from the antenna origin along reflectometer the line of sight.

Both reconstructions are able to track the separatrix radial scans at higher density layers. The magnetic field variation starting at 3.5 seconds does not influence either density profile reconstructions, as is expected.

The shape of the outer density layers shows higher differences between reconstructions than the layers further inside the plasma, indicating a high sensitivity of the outer edge density profile reconstruction to the FF measurement. 


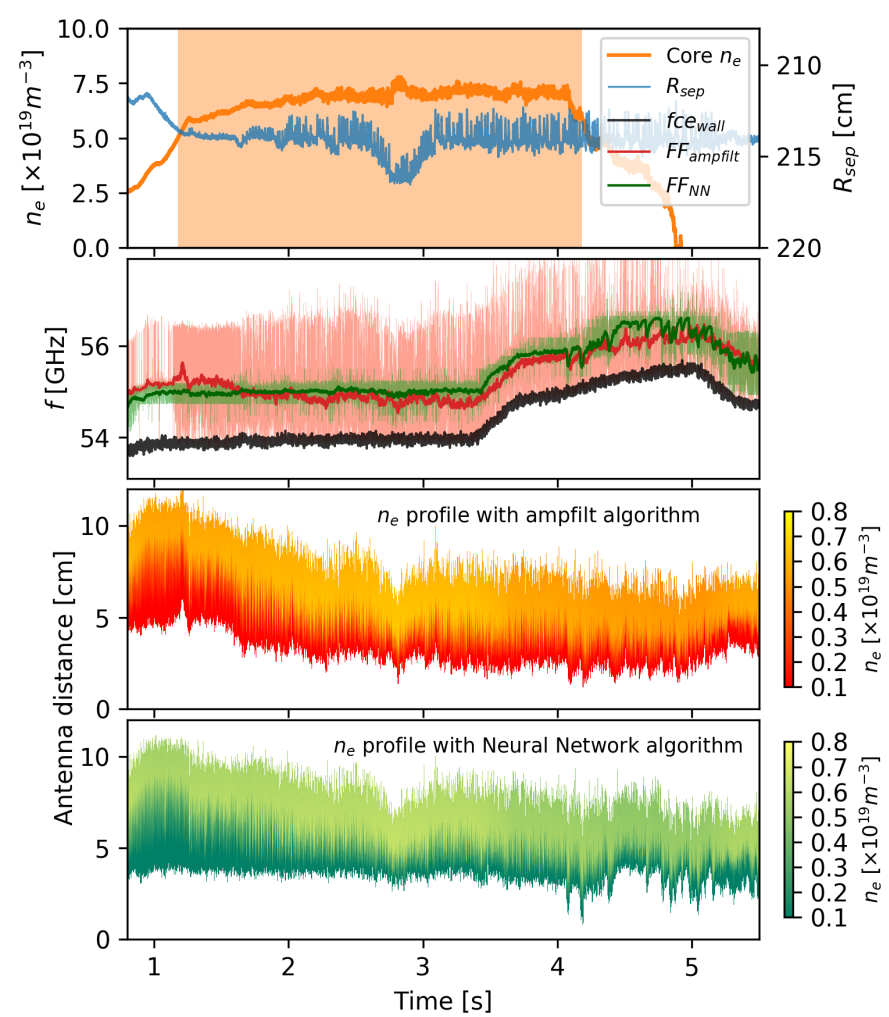

Fig. 11. Reconstructed density profiles for AUG\#33841 using the ampfilt and neural network algorithms for first fringe frequency estimation.

The neural network reconstruction shows an approximately constant vacuum distance, between the reflectometry antennas and the first plasma reflection layer, throughout the discharge. This can be expected since the reflectometry antenna is embedded in the shadow but very close to the ICRF antenna limiters.

\section{CONCLUSION}

In this work we detailed the developments made to improve the error of the group delay measurement of the new ICRF embedded X-mode multichannel edge density profile reflectometry diagnostic on ASDEX Upgrade. The group delay measurement and resulting radial accuracy of the reconstructed density profiles are directly dependent on a good first fringe frequency estimation.

To improve the automatic processing of complete discharges using the ampfilt algorithm, a neural network approach for FF estimation was implemented. The implemented NN was trained with experimental data to estimate the first fringe frequency in a broad range of operational conditions from the raw spectrogram data.

Both FF estimation methods were compared based on a dataset with known FF frequency values. However, it is important to acknowledge that the evaluation of the FF estimation error may still be biased as there is no known true FF value for comparison. The training and validation dataset was created by experienced X-mode diagnosticians, which have an unknown estimation error and biasing. This may be improved by averaging the same FF estimations made by different diagnosticians.

We were able to improve the error and precision of FF estimation using the new neural network approach when compared to the previous ampfilt algorithm. The density profile reconstruction using the neural network approach showed seemingly more accurate results than the previous method using the ampfilt algorithm. Still, the evaluation of density profile reconstruction error requires comparison with other edge electron density profile diagnostic reconstructions and associated radial and density errors.

The improved FF estimation results show that neural network techniques may provide more interesting and improved results than other computational algorithms for issues where there is no known solution.

\section{ACKNOWLEDGMENT}

This work has been carried out within the framework of the EUROfusion Consortium and has received funding from the Euratom research and training programme 20142018 under grant agreement No 633053. IST activities also received financial support from Fundação para a Ciência e Tecnologia through project UID/FIS/50010/2013 and the PhD Scholarship FCT-SFRH/BD/52414/2013. The views and opinions expressed herein do not necessarily reflect those of the European Commission.

\section{REFERENCES}

[1] D. E. Aguiam, A. Silva, V. Bobkov, P. J. Carvalho, P. F. Carvalho, R. Cavazzana, G. D. Conway, O. D’Arcangelo, L. Fattorini, H. Faugel, A. Fernandes, H. Fünfgelder, B. Gonçalves, L. Guimarais, G. De Masi, L. Meneses, J. M. Noterdaeme, R. C. Pereira, G. Rocchi, J. M. Santos, A. A. Tuccillo, and O. Tudisco, "Implementation of the new multichannel X-mode edge density profile reflectometer for the ICRF antenna on ASDEX Upgrade," Review of Scientific Instruments, vol. 87, no. 11, p. 11E722, nov 2016. [Online]. Available: http://aip.scitation.org/doi/10.1063/1.4961558

[2] W. Zhang, V. Bobkov, T. Lunt, J.-M. Noterdaeme, D. Coster, R. Bilato, P. Jacquet, D. Brida, Y. Feng, E. Wolfrum, and L. Guimarais, "3D simulations of gas puff effects on edge density and ICRF coupling in ASDEX Upgrade," Nuclear Fusion, vol. 56, no. 3, p. 036007, mar 2016. [Online]. Available: http://stacks.iop.org/0029$5515 / 56 / \mathrm{i}=3 / \mathrm{a}=036007$ ?key=crossref.5fd13606a657c6ad866987fc137798bd

[3] E. Mazzucato, "Microwave reflectometry for magnetically confined plasmas," Review of Scientific Instruments, vol. 69, p. 2201, 1998.

[4] K. W. Kim, E. J. Doyle, W. A. Peebles, A. Ejiri, N. C. Luhmann, and C. L. Rettig, "Advances in reflectometric density profile measurements on the DIII-D tokamak," Review of Scientific Instruments, vol. 66, no. 2, pp. 1229-1232, 1995.

[5] F. Clairet, C. Bottereau, J. M. Chareau, M. Paume, and R. Sabot, "Edge density profile measurements by X-mode reflectometry on Tore Supra,' Plasma Physics and Controlled Fusion, vol. 43, no. 4, p. 429, 2001.

[6] H. Qu, T. Zhang, S. Zhang, F. Wen, Y. Wang, D. Kong, X. Han, Y. Yang, Y. Gao, C. Huang, J. Cai, and X. Gao, "Q-Band X-Mode Reflectometry and Density Profile Reconstruction," Plasma Science and Technology, vol. 17, no. 12, pp. 985-990, 2015.

[7] P. J. Carvalho, L. Meneses, J. Santos, B. Gonçalves, and J. Contributors, "A hybrid X-mode reflectometry first fringe detection algorithm for JET," 1st EPS conference on Plasma Diagnostics (ECPD2015), no. 633053, 2015.

[8] C. Lau, G. Hanson, Y. Lin, J. Wilgen, S. Wukitch, B. Labombard, and G. Wallace, "First results of the SOL reflectometer on Alcator C-Mod," Review of Scientific Instruments, vol. 83, no. 10, pp. 1-4, 2012.

[9] G. Wang, L. Zeng, E. J. Doyle, T. L. Rhodes, and W. A. Peebles, "Improved reflectometer electron density profile measurements on DIIID," Review of Scientific Instruments, vol. 74, no. 3 II, pp. 1525-1529, 2003. 
[10] D. Aguiam, A. Silva, V. Bobkov, P. Carvalho, P. Carvalho, R. Cavazzana, G. Conway, O. D'Arcangelo, L. Fattorini, H. Faugel, A. Fernandes, H. Fünfgelder, B. Goncalves, L. Guimarais, G. De Masi, L. Meneses, J. Noterdaeme, R. Pereira, G. Rocchi, J. Santos, A. Tuccillo, and O. Tudisco, "X-mode raw data analysis of the new AUG ICRF antenna edge density profile reflectometer," Fusion Engineering and Design, vol. 87, no. 11, pp. 7-10, apr 2017. [Online]. Available: http://linkinghub.elsevier.com/retrieve/pii/S0920379617304222

[11] Y. LeCun, Y. Bengio, and G. Hinton, "Deep learning," Nature, vol. 521, no. 7553, pp. 436-444, may 2015. [Online]. Available: http://arxiv.org/abs/1603.05691 http://www.nature.com/doifinder/10.1038/nature14539

[12] A. Agarwal, P. Barham, E. Brevdo, Z. Chen, C. Citro, G. S Corrado, A. Davis, J. Dean, M. Devin, S. Ghemawat, I. Goodfellow, A. Harp, G. Irving, M. Isard, Y. Jia, R. Jozefowicz, L. Kaiser, M. Kudlur, J. Levenberg, D. Man, R. Monga, S. Moore, D. Murray, C. Olah, M. Schuster, J. Shlens, B. Steiner, I. Sutskever, K. Talwar, P. Tucker, V. Vanhoucke, V. Vasudevan, F. Vi, O. Vinyals, P. Warden, M. Wattenberg, M. Wicke, Y. Yu, and X. Zheng, "TensorFlow: Large-Scale Machine Learning on Heterogeneous Systems," White Paper, 2015. [Online]. Available: http://tensorflow.org/

[13] F. Chollet, "Keras: Deep Learning library for Theano and TensorFlow," GitHub Repository, pp. 1-21, 2015.

[14] — - Deep Learning with Python. Shelter Island, NY: Manning Publications Co., 2017, vol. 1.

[15] F. A. Matos, D. R. Ferreira, and P. J. Carvalho, "Deep learning for plasma tomography using the bolometer system at JET," Fusion Engineering and Design, vol. 114, pp. 18-25, 2017.

[16] O. Barana, A. Murari, I. Coffey, and JET-EFDA Contributors, "Artificial Neural Networks for Real-time Diagnostic of High-Z Impurities in Reactor-relevant Plasmas," in 2007 IEEE International Symposium on Intelligent Signal Processing. IEEE, 2007, pp. 1-5. [Online]. Available: http://ieeexplore.ieee.org/document/4447609/

[17] J. Santos, F. Nunes, M. Manso, and I. Nunes, "Neural network evaluation of reflectometry density profiles for control purposes," Review of Scientific Instruments, vol. 70, no. 1, p. 521, 1999. [Online]. Available: http://ieeexplore.ieee.org/xpl/articleDetails.jsp?arnumber=4996359

[18] J. Santos, "Fast reconstruction of reflectometry density profiles on ASDEX Upgrade for plasma position feedback purposes," Ph.D. dissertation, Instituto Superior Técnico, Universidade Técnica de Lisboa, 2008.

[19] J. Hernandez, A. Vannucci, T. Tajima, Z. Lin, W. Horton, and S. McCool, "Neural network prediction of some classes of tokamak disruptions," Nuclear Fusion, vol. 36, no. 8, pp. 1009-1017, 1996. [Online]. Available: http://stacks.iop.org/0029$5515 / 36 / \mathrm{i}=8 / \mathrm{a}=\mathrm{I} 05$ ? $\mathrm{key}=$ crossref.932af685217f157eb23aed83dd6e 3550

[20] C. Windsor, G. Pautasso, C. Tichmann, R. Buttery, T. Hender, J. E. Contributors, and t. A. U. Team, "A cross-tokamak neural network disruption predictor for the JET and ASDEX Upgrade tokamaks," Nuclear Fusion, vol. 45, no. 5, pp. 337-350, 2005. [Online]. Available: http://stacks.iop.org/00295515/45/i=5/a=004?key=crossref.170e4cfeab7836eaf $142634 \mathrm{f} 3 \mathrm{e} 851578$

[21] D. P. Kingma and J. Ba, "Adam: A Method for Stochastic Optimization," pp. 1-15, dec 2014. [Online]. Available: http://arxiv.org/abs/1412.6980

[22] R. E. Kalman, "A New Approach to Linear Filtering and Prediction Problems," Journal of Basic Engineering, vol. 82, no. 1, p. 35, 1960. [Online]. Available: http://fluidsengineering.asmedigitalcollection.asme.org/article. aspx? articleid=1430402 